\title{
Estratégia de enriquecimento com Cereus jamacaru DC. em área de clareira introduzido por propagação vegetativa
}

\author{
Enrichment strategy with Cereus jamacaru DC. in a clearing area introduced by vegetative \\ propagation \\ Estrategia de enriquecimiento con Cereus jamacaru DC. en un área de desmonte introducida por \\ propagación vegetativa
}

Iracy Amélia Pereira Lopes ORCID: https://orcid.org/0000-0003-1641-6214 Universidade Federal de Campina Grande, Brasil E-mail: iracyamelia.lopes@gmail.com

Adriano Salviano Lopes

ORCID: https://orcid.org/0000-0002-3182-3478 Universidade Federal da Paraíba, Brasil E-mail: adrianolopes5656@gmail.com

Khyson Gomes Abreu

ORCID: https://orcid.org/0000-0002-3439-6598 Universidade Federal da Paraíba, Brasil E-mail: khyson-cunha@hotmail.com

Rosilvam Ramos de Sousa

ORCID: https://orcid.org/0000-0002-3770-2992 Universidade Federal de Campina Grande, Brasil E-mail: rosilvam17@gmail.com

Alecksandra Vieira de Lacerda ORCID: https://orcid.org/0000-0002-9703-3997 Universidade Federal de Campina Grande, Brasil E-mail: alecvieira@yahoo.com.br

Iara Almeida Roque ORCID: https://orcid.org/0000-0002-7807-3301 Universidade Federal de Campina Grande, Brasil E-mail: yara.roque.sb@gmail.com

Iracema de Azevedo Monte Paiva ORCID: https://orcid.org/0000-0002-3246-2280

Universidade Estadual da Paraíba, Brasil E-mail: iracemapaiva1997@gmail.com

\begin{abstract}
Resumo
O mandacaru (Cereus jamacaru DC) é uma espécie endêmica da caatinga que se encontra em extinção devido a ação antrópica por meio da pratica da agropecuária extrativista, desmatamento, queimadas entre outras.A pesquisa objetivou avaliar as taxas de sobrevivência e de desenvolvimento de Cereusjamacaru DC. Introduzido por propagação vegetativa em áreas de clareira no semiárido paraibano.Foi realizado o plantio de223 cladódios em três clareiras no Espaço Experimental Reservado para Estudos de Ecologia e Dinâmica da Caatinga - Área II, dispostos em linhas no espaçamento de $1 \mathrm{~m}$ entre plantas e $2 \mathrm{~m}$ entre linhas. Realizou-se a medição detamanho e diâmetro dos cladódiose o monitoramento mensal da sobrevivência e das fenofases reprodutivas, correlacionado com os dados de precipitação para o período de janeiro a julho de 2017. Não ocorreu mortalidade nos primeiros meses seguintes ao plantio, mas somente a partir do mês de maio, quando foi registrado o pico com 33 indivíduos, sendo observada mortalidade ainda no mês de julho, quando se registrou a morte de nove indivíduos, perfazendo um percentual de $18,8 \%$ do total de cladódios implantados. De modo geral, C. jamacaru apresentou-se viável para plantio em área de clareira, podendo se tornar uma alternativa de cultivo para os agricultores em suas propriedades, servindo na recuperação de áreas degradadas.
\end{abstract}

Palavras-chave: Lavoura xerófila; Ecologia populacional; Sistemas naturais; Região semiárida.

\section{Abstract}

Mandacaru (Cereus jamacaru DC) is an endemic species of the caatinga that is endangered due to anthropic action through the practice of extractive agriculture, deforestation, fires, among others. The research aimed to evaluate the survival and development rates of Cereus jamacaru DC. introduced by vegetative propagation in clearing areas in the 
semi-arid region of Paraíba. 223 cladodes were planted in three clearings in the Experimental Space Reserved for Studies of Ecology and Dynamics of the Caatinga - Area II, arranged in lines spaced $1 \mathrm{~m}$ between plants and $2 \mathrm{~m}$ between lines. Cladodes size and diameter were measured and monthly monitoring of reproductive survival and phenophases was correlated with precipitation data for the period from January to July 2017. There was no mortality in the first months after planting, but only from the month of May, when the peak was registered with 33 individuals, with mortality still being observed in the month of July, when the death of nine individuals was registered, making up a percentage of $18.8 \%$ of the total implanted cladodes. In general, C. jamacaru proved to be viable for planting in a clearing area, and could become an alternative crop for farmers on their properties, serving in the recovery of degraded areas.

Keywords: Xerophile agriculture; Population ecology; Natural systems; Semi-arid region.

\section{Resumen}

Mandacaru (Cereus jamacaruDC) es una especieendémica de la caatinga que se encuentraenpeligro de extincióndebido a la acciónantrópica a través de la práctica de agriculturaextractiva, deforestación, incendios, entre otros. La investigacióntuvocomoobjetivoevaluar las tasas de supervivencia y desarrollo de Cereus jamacaru DC. introducido por propagaciónvegetativaenáreas de desmonte de la regiónsemiárida de Paraíba. Se plantaron 223 cladodiosentres claros del Espacio Experimental Reservado para Estudios de Ecología y Dinámica de la Caatinga - Área II, dispuestosenlíneasespaciadas $1 \mathrm{~m}$ entre plantas y $2 \mathrm{~m}$ entre líneas. Se midió el tamaño y diámetro de los cladodios y se correlacionó el monitoreomensual de supervivenciareproductiva y fenofases con los datos de precipitación para el período de enero a julio de 2017. No hubomortalidaden los primerosmesesdespués de la siembra, sino solo a partir del mes de mayo, cuando el pico se registró con 33 individuos, observándosemortalidadaúnen el mes de julio, cuando se registró la muerte de nueveindividuos, lo que representó un porcentaje del 18,8\% del total de cladodiosimplantados. En general, C. jamacarudemostró ser viable para la siembraen un área de desmonte, y podríaconvertirseen un cultivo alternativo para los agricultoresen sus propiedades, sirviendoen la recuperación de áreasdegradadas.

Palabras clave: Cultivoxerófilo; Ecología de la población; Sistemas naturales; Región semiárida.

\section{Introdução}

O Semiárido brasileiro se estende por todos os estados da região Nordeste mais o norte de Minas Gerais. Totalizando uma extensão territorial de $1.127 .953 \mathrm{~km}^{2}$ distribuídos em 1.262 municípios, tendo cerca de 27.870.241 habitantes. A maior parte do total de municípios é considerada de pequeno porte, e onde residem $65 \%$ da população total do Semiárido, enquanto nos de médio porte $17 \%$ e nos de grande porte $18 \%$ (Medeiros, 2018).

De acordo com o MMA (2017), as áreas relativas ao Bioma Caatinga abrangem cerca de 10\% do território nacional, sendo o principal ecossistema da região Nordeste brasileiro. Esse Bioma é caracterizado pelo elevado número de espécies adaptadas a longos períodos de estiagem. Entretanto, trata-se de uma área pouco conhecida, no que se referem as suas riquezas biológicas, devido à carência de pesquisas detalhadas sobre os potenciais na região.

A Caatinga é classificada como savana estépica e é o terceiro Bioma mais degradado do Brasil, perdendo apenas para a Floresta Atlântica e o Cerrado (Souza et al., 2015). Esses autores estimam que $80 \%$ da vegetação encontram-se completamente modificada, devido às ações de extrativismo e da agropecuária.

Os recursos vegetais oriundos da Caatinga contribuem diretamente para a sobrevivência das populações rurais existentes no seu meio, uma vez que disponibilizam diversos subsídios, fornecendo os recursos que auxiliam e contribuem para qualidade de vida dos povos do Semiárido (Almeida et al., 2006).

Diante de tantas preocupações ambientais, e com o objetivo de evitar a degradação dos recursos naturais disponíveis, tem-se destacado a importância da implantação de ações como, por exemplo, enriquecimento da Caatinga, como sendo uma alternativa de alcance de renda e uso sustentável nos ecossistemas naturais (Lima, 2016).

Dentre essas espécies nativas da Caatinga, o mandacaru (Cereus jamacaru DC.), considerado uma planta símbolo do nordeste brasileiro, apresenta um enorme potencial econômico devido a sua rusticidade e ser bem adaptado ao Bioma, resistindo a longos períodos de estiagem, e muito utilizado como forrageira, servindo de alimento para o rebanho em períodos mais seco do ano (Andrade-Lima, 1981). Devido a esse fato acredita-se que o enriquecimento de áreas de Caatinga com a introdução desta 
espécie é uma opção, que deve ser replicada pelos sertanejos, para que venha melhorar as condições sociais e econômicas, além de amenizar os impactos negativos no meio ambiente.

É inegável o potencial dessa cactácea para a recuperação de áreas degradadas. Portanto, é necessário que se promova métodos de uso, manejo e conservação da espécie associadas a ambientes de clareiras, onde essa prática possa contribuir para o estabelecimento em áreas de Caatinga. Desta forma, essa pesquisa objetivou-se avaliar as taxas de sobrevivência e de desenvolvimento de C.jamacaru introduzido por propagação vegetativa em áreas de clareira no Semiárido paraibano.

\section{Metodologia}

A pesquisa foi realizada em três áreas de clareiras dentro do Espaço Experimental Reservado para Estudos de Ecologia e Dinâmica da Caatinga - Área II, pertencente ao Laboratório de Ecologia e Botânica - LAEB/CDSA/UFCG (7³9'42.03" S e 36 $53^{\circ} 46.61$ " W; $528 \mathrm{~m}$ de altitude) no município de Sumé-PB, durante os meses de janeiro a julho de 2017. O clima da região é classificado por Köppen como BSh (Alvares et al., 2013). Apresenta período chuvoso de fevereiro a março, com uma precipitação média de $585 \mathrm{~mm}$ e uma temperatura média de $24{ }^{\circ} \mathrm{C}$ (AESA, 2020).

Foram identificadas as três áreas de clareira dentro da área experimental e posteriormente, houve a seleção dos indivíduos adultos de $C$. jamacaru na área rural do município de Sumé para a retirada de cladódios, os mesmos foram divididos em seis classes de tamanho (21-30; 31-40; 41-50;51-60; 61-70; 71-80 cm) e quatro classes diamétricas (3-5; 6-8; 9-11; 12-14 cm).

O plantio foi efetuado no mês de janeiro de 2017 após a abertura dos berços com profundidade de $15 \mathrm{~cm}$. Assim, as partes vegetativas do $C$. jamacaru foram dispostas em linhas no espaçamento de $1 \mathrm{~m}$ entre plantas e $2 \mathrm{~m}$ entre linhas, perfazendo um total de 223 cladódios distribuídos de acordo com o tamanho das clareiras (Clareira 1-75; Clareira 2-101; Clareira 3-47 cladódios).

$\mathrm{O}$ acompanhamento do desenvolvimento e sobrevivência deste material introduzido foi realizado mensalmente, com substituição dos indivíduos mortos, realizando ainda a verificação de surgimento de órgãos reprodutivo (frutos e flores) nos cladódios após o plantio. Efetuou-se também a substituição das mudas que não sobreviveram, permitindo uma avaliação contínua das estratégias ecológicas de enriquecimento.

Os dados climáticos foram obtidos diariamente a partir da estação meteorológica mais próxima do local selecionado, visando obter o comportamento dos elementos climáticos e sua influência sobre as plantas.

Utilizaram-se para a tabulação de dados o programa Microsoft Office Excel, versão 2010 e a apresentação dos dados foi feita em forma de gráficos.

\section{Resultados e Discussão}

A maior parte dos cladódios de C. jamacaru faz parte da classe de tamanho entre 41 e $50 \mathrm{~cm}$, contendo cerca de $62 \%$ dos exemplares (Figura 1). Esse tamanho de cladódio foi o mesmo utilizado por Guedes et al. (2018) ao trabalharem com mandacaru com e sem espinhos em área de Caatinga raleada. Como também por Silva et al. (2011) e Silva et al. (2012), durante o estudoda influência da densidade no desenvolvimento doPilosocereusgounellei e do C. jamacaru, respectivamente. 
Figura 1 - Distribuição em classes de tamanho dos cladódios de $C$. jamacaru utilizados em plantio de enriquecimento em área de Caatinga no Cariri Ocidental da Paraíba.

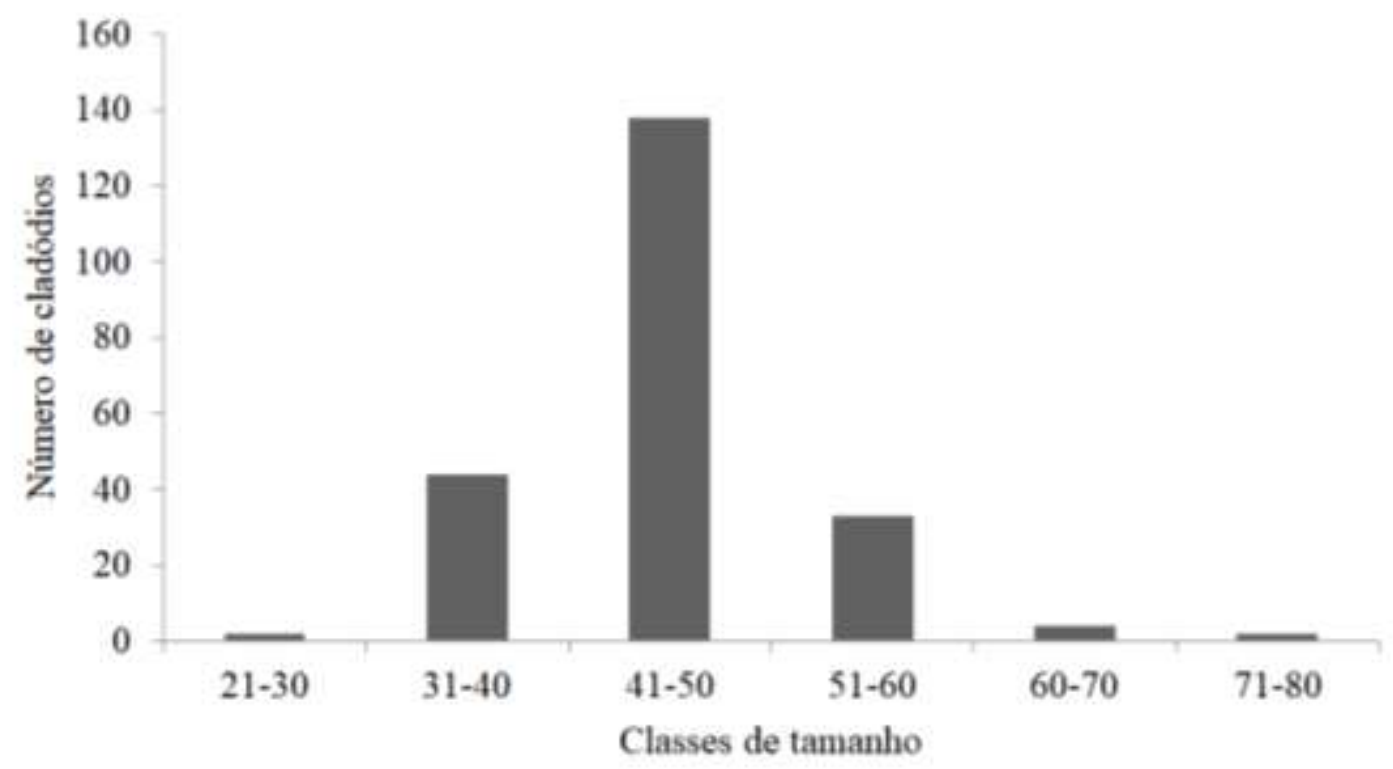

Fonte: Dados da Pesquisa.

Já Correia et al. (2011), estudando uma variedade sem espinho dessa mesma espécie, usaram estacas de $30 \mathrm{~cm}$, o que por sua vez facilita o seu manuseio. Por outro lado, quanto mais desenvolvido for o cladódio, maior suas reservas e consequentemente, mais preparado para sobreviver as condições de campo.

Uma vantagem de usar cladódios em áreas degradadas é fato de poder antecipar algumas etapas no desenvolvimento do indivíduo, como o relatado por Correia et al. (2012), que avaliaram a produção a partir das sementes retiradas do fruto e verificaram que aos 90 dias após o início da germinação, as plântulas apresentavam altura em torno de 3,0 cm. Tendo em vista o tempo necessário para as plantas obterem essa altura, é muito mais interessante adotar alternativas, a exemplo do plantio dos cladódios, visando acelerar o processo, uma vez que os mesmos são plantados com a altura média de $50 \mathrm{~cm}$, o que torna os indivíduos menos vulneráveis em campo.

Relacionado ao diâmetro, a quase totalidade dos indivíduos se concentrou nas classes6-8cm (35\%) e 9-11cm (62\%) (Figura 2), essas classes apresentam diâmetros médioscom uma quantidade considerável de nutrientes e água. Até certo ponto essa característica é desejável, pois ajuda na sobrevivência durante esse primeiro período de maior vulnerabilidade.

Segundo Guedes et al. (2018), um maior nível de hidratação pode favorecer a morte dos indivíduos de $C$. jamacaru mesmo com o local do corte totalmente cicatrizado. Silva et al.(2007) considera que as cactáceas devem ser plantadas no final da estação seca, pois elas irão apresentar menor quantidade de água em seu tecido o que ajuda na fixação das estacas nos berços e evita o apodrecimento. 
Figura 2 - Distribuição em classes de diâmetro dos cladódios de $C$. jamacaru utilizados em plantio de enriquecimento em uma área de Caatinga no Cariri Ocidental da Paraíba.

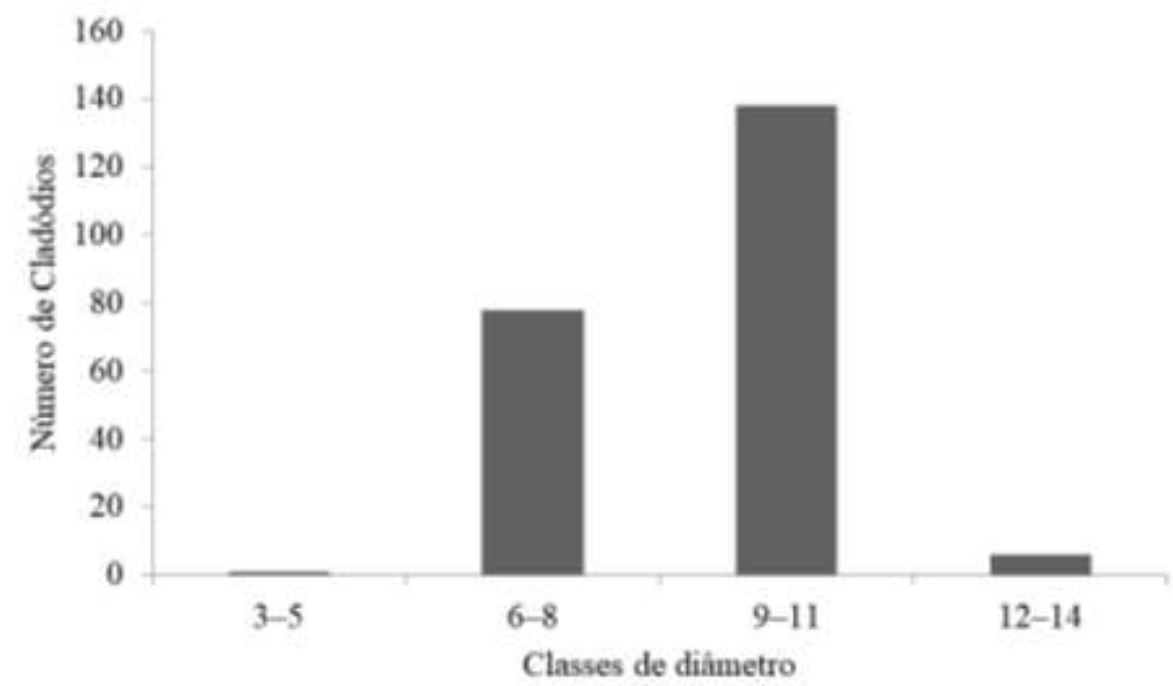

Fonte: Dados da Pesquisa.

Os dados obtidos nesse estudo, referente à análise de sobrevivência dos indivíduos de C. jamacaru, demonstraram que 81,2\% dos cladódios sobreviveram ao período do experimento, com a mortalidade ocorrendo somente a partir do quarto mês (maio), quando foi registrado o pico de morte com 33 cladódios, sendo observada mortalidade ainda no último mês de avaliação (julho), quando se registrou a morte de outros nove indivíduos (Figura 3). Resultados semelhantes foram relatados por Silva et al. (2012), no qual registraram 91,66\% de sobrevivência dessa espécie. Guedes et al. (2018) também observaram uma sobrevivência acima de 90\% até os dois meses de idade, porém essa sobrevivência diminuiu com o passar do tempo, chegando a 10\% após 36 meses.

Figura 3 - Mortalidade de cladódios de C. jamacaru em plantio de enriquecimento da Caatinga associado à variação da precipitação dos sete meses iniciais do ano de 2017 e a precipitação média histórica para o município de Sumé-PB.

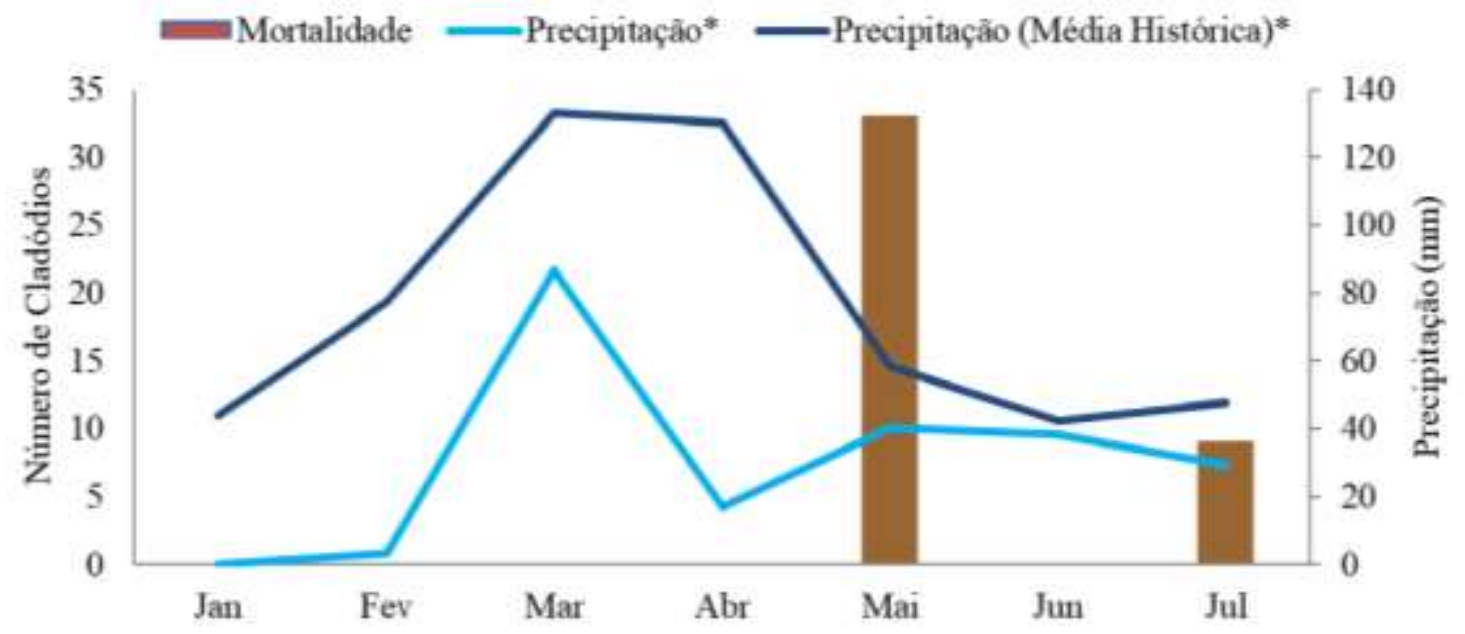

Fonte: Adaptado de AESA (2017). 
No período de implantação, ou seja, de fevereiro a abril, o total acumulado foi de 107,2 mm, porém a distribuição foi bastante irregular, com ocorrência de chuvas concentradas no mês de março, quando se registrou mais de $80 \%$ do total precipitado $(86,9 \mathrm{~mm})$. Assim, a mortalidade das plantas pode ter ocorrido como resposta à variação da precipitação, que influenciou fortemente na sobrevivência dos indivíduos.

Segundo Souto et al. (2017), a água é um elemento muito importante na produção vegetal, e a sua oferta e manejo correto é de suma importância para o desenvolvimento e sobrevivência das plantas. Silva et al. (2007) destaca a capacidade e eficiência que as cactáceas têm para o armazenamento de água, entretanto a pesquisa indica a importância da disponibilidade hídrica para a sobrevivência dos indivíduos no período inicial da implantação em campo.

Por ser uma espécie adaptada às condições de limitações hídricas, $C$ jamacaru possui células parenquimáticas especializadas em armazenar água e grandes proporções de tecido parenquimático com células constituídas por mucilagem, adaptações estas que também proporcionam grande resistência a secas prolongadas (Dettke\&Milaneze-Gutierre, 2008). Taylor e Zappi (2004) citam as adaptações morfofisiológicas específicas da espécie, a exemplo da presença de caule fotossinteticamente ativo e sistemas radiculares superficiais e extensos, que permitem uma rápida absorção de água depois de uma seca prolongada.

Assim, os resultados podem ser um indicativo de que o baixo volume e irregularidade da precipitação, no período de implantação, influenciaram na sobrevivência das plantas, pois apesar de ser uma espécie adaptada às condições climáticas da região Semiárida, os indivíduos de C. jamacaru sofrem com os longos períodos de estiagem, o que pode resultar na diminuição de sua população na Caatinga.

Com relação à fenologia reprodutiva, tem-se que os cladódios começaram a expressar as fenofases no mês de maio, quando foi registrada a floração de 33 indivíduos e frutificação de 21 cladódios. Para o mês de junho observou-se o decréscimo no número de indivíduos expressando floração (14) e frutificação (três). A dispersão só foi verificada no mês de julho, em seis indivíduos (Figura 4).

Analisando a ocorrência das fenofases e a precipitação registrada no período, tem-se que no mês de maio, quando ocorreram os picos de floração e frutificação, já no final do período chuvoso, a precipitação ainda foi expressiva, e se manteve constante e bem distribuída também no mês de junho, quando houve um decréscimo da frutificação e iniciou a dispersão dos frutos existentes.

Figura 4 - Fenofases de cladódios de $C$. jamacaru em plantio de enriquecimento da Caatinga associado à variação da precipitação dos sete meses iniciais do ano de 2017 e a precipitação média histórica para o município de Sumé-PB.

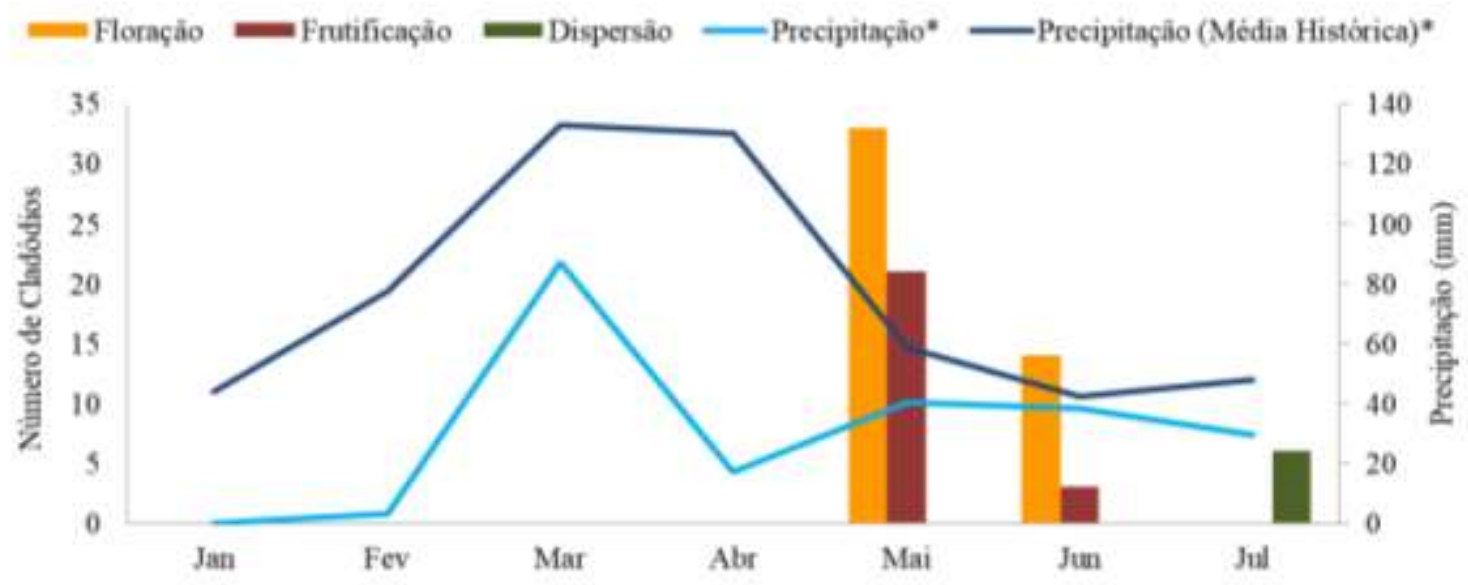

Fonte: Adaptado de AESA (2017). 
Lima (2007) destaca em seu trabalho, que mesmo em ambientes seco, podem ocorrer diferentes padrões fenológicos, o que pode ser definido, principalmente pela chuva, ou pela eficiência da planta em ganhar e armazenar água. Taylor e Stuppy (2004) relacionaram os eventos de floração e frutificação de $C$. jamacaru e outras cactáceas com a precipitação, e concluíram que, com exceção de Tacingapalmadora, as outras espécies estudadas não apresentaram correlação com a precipitação, sendo que a maioria floresceu e frutificou no final da estação seca e início da estação chuvosa.

Morellato e Leitão Filho (1992) discutem que os padrões de floração sazonais, observados nos ambientes tropicais, é uma resposta à alternância da estação seca e chuvosa, considerada como o principal fator desencadeador das fenofases.

Além disso, é evidente que os fatores ambientais influenciam o desenvolvimento das espécies. Zanine e Santos (2004) enfatizam em seu trabalho, que plantas podem competir entre si e com outras plantas pelos recursos do meio (luz, água, nutrientes, CO2 etc.), e o tempo de duração da competição determina prejuízos no crescimento, no desenvolvimento e, consequentemente, na produção das plantas.

De modo geral, verificou-se que, apesar dos cladódios serem partes de um indivíduo adulto, e, portanto, expressarem os eventos reprodutivos, um baixo percentual de indivíduos expressaram as fenofases, provavelmente em decorrência de limitações morfofisiológicas resultantes da adaptação, para o desenvolvimento e formação de um novo indivíduo.

\section{Conclusão}

De modo geral, o Cereus jamacaru DC. Apresentou-se viável para plantio em área de clareira, uma vez que a espécie possui adaptações para solos degradados.

Essa espécie pode se tornar uma alternativa de cultivo para os agricultores em suas propriedades, pois representa um componente biológico de ocupação de espaços que pode auxiliar na recuperação de áreas, além de servir como fonte de alimento para o homem e animais silvestres, e ser uma importante forrageira para os rebanhos no período seco.

\section{Referências}

Agência Executiva de Gestão das Águas do Estado da Paraíba. (2017). Meteorologia: chuvas. <http://www.aesa.pb.gov.br/aesa-website/meteorologiachuvas/?formdate $=2020-01-28 \&$ produto $=$ municipio \&periodo $=$ anual $>$.

Agência Executiva de Gestão das Águas do Estado da Paraíba. (2020). Precipitação máxima dos municípios/postos no ano 2020. <http://www.aesa.pb.gov.br/aesa-website/meteorologia-chuvas/?formdate=2020-01-28\&produto=municipio\&periodo=anual > .

Almeida, C. F. B. R., Amorim, E. L. C., Albuquerque, U. P., \& Maia, M. B. (2006). Medicinal plants popularly used in the Xingó region - a Semi-arid location in Northeastern Brazil. Journal of Etnobiology and Ethnomedicine, 2 (15), 1-7. 10.1186/1746-4269-2-15.

Alvares, C. A., Stape, J. L., Sentelhas, P. C., Gonçalves, J. L. M., \& Sparovek, G. Köppen’s(2013). climate classification map for Brazil. MeteorologischeZeitschrift, 22 (6), 711-728. 10.1127/0941-2948/2013/0507.

Andrade-Lima, D. (1981). The Caatingas dominium.Revista Brasileira de Botânica, 4, 149-153.

Correia, D., Nascimento, E. H. S., Araújo, J. D. M., \& Oliveira, A. E. R. (2011). Propagação de mandacaru sem espinhos.[Embrapa Agroindústria Tropical]. https://ainfo.cnptia.embrapa.br/digital/bitstream/item/107305/1/BPD11013.pdf.

Correia, D., Silva, I. C., Nascimento, E. H. S., Morais, J. P. S. (2012). Produção de Mudas de Mandacaru.[Embrapa Agroindústria Tropical]. https://www.infoteca.cnptia.embrapa.br/bitstream/doc/951853/1/CIT12002.pdf.

Dettke, G. A., \& Milaneze-Gutierre, M. A. (2008). Anatomia caulinar de espécies epífitas de Cactaceae, subfamília Cactoideae. Hoehnea, 35 (1), 583-595. https://doi.org/10.1590/S2236-89062008000400010.

Guedes, F. L., Silva, N. L., Souza, H. A., \& Pompeu, R. C. F. F. (2018). Cultivo de mandacaru (Cereusspp) em Caatinga raleada. [Embrapa Caprinos e Ovinos]. https://ainfo.cnptia.embrapa.br/digital/bitstream/item/200326/1/CNPC-2018-COT-181.pdf.

Lima, A. L. A. (2007). Padrões fenológicos de espécies lenhosas e cactáceas em uma área do semiárido do Nordeste do Brasil. Recife, Dissertação (Mestrado em Botânica) - Universidade Federal Rural de Pernambuco- UFRPE, Pernambuco, PE, Brasil.

Lima, L. H. C. (2016). Avaliação de enriquecimento da Caatinga com mudas enxertadas de Umbuzeiro (Spondias tuberosa Arruda Cam) em uma área no semiárido paraibano, Brasil.Sumé, Monografia (Tecnólogo em Agroecologia)- Universidade Federal de Campina Grande -UFCG, Paraíba, PB, Brasil. 
Research, Society and Development, v. 10, n. 2, e24210212458, 2021

(CC BY 4.0) | ISSN 2525-3409 | DOI: http://dx.doi.org/10.33448/rsd-v10i2.12458

Medeiros, S. S. Agricultura temporária. (2018). [Instituto Nacional do Semiárido]. https://portal.insa.gov.br/images/acervolivros/Tabela\%20Agricultura_RESUMO\%203.pdf.

Ministério do Meio Ambiente. (2017). Caatinga: contexto, características e estratégias de conservação [Blog]. http://www.mma.gov.br/biomas/caatinga/item/191.

Morellato, L. P. C., \& Leitão Filho, H. F. Padrões de frutificação e dispersão na Serra do Japi. In: Morellato, L. P. C. (Org.). (1992). História natural da Serra do Japi: ecologia e preservação de uma área florestal no Sudeste do Brasil: Editora da Unicamp/Fapesp, (112-140).

Silva, J. G. M., Lima, J. F. C., \& Maciel, F. C. (2007). Utilização e manejo do Xiquexique e Mandacaru como reservas estratégicas de forragem. Natal:Empresa de Pesquisa Agropecuária do Rio Grande do Norte S/A - EMPARN.

Silva, J. G. M., Melo, S. S. N. S., Diniz, M. C. N. M., Medeiros, M. R., Silva, S. Y. A. M., \& Araújo, M. S. (2012) Características Morfofisiológicas e Produção do Mandacaru Cultivado em Diferentes Densidades. Revista Centauro, 3 (1), 33-43, http://crmvrn.gov.br/documents/revista/vol3/caracteristicas_morfofisiologicas_e_producao_mandacaru_cultivado_em_diferentes_densidades.pdf.

Silva, J. G. M., Silva, D. S., Pereira, W. E., Diniz, M. C. N. M., Silva, G. J. A. M., \& Medeiros, M. R. (2011). Características Morfofisiológicas e Produção do Xiquexique Cultivado em Diferentes Densidades. Revista Centauro, 2 2 (1), http://crmvrn.gov.br/documents/revista/vol2/Rev\%20Centauro\%20v2\%20n1\%2008-17-1.pdf.

Souto, J. S., Nascimento Neto, J. H., Leonardo, F. A. P., Souto, P. C., Borges, C. H. A. (2017) Uso da técnica restauradora "BOCAJ" em áreas de Caatinga no Seridó da Paraíba, Brasil. Revista Agropecuária científica no Semiárido, 13 (2), 154-161. http://dx.doi.org/10.30969/acsa.v13i2.899.

Souza, B. I., Artigas, R. C., \& Lima, E. R. V. (2015). Caatinga e desertificação. RevistaMercador, 14 (1), 131-150. doi: 10.4215/RM2015.1401. 0009.

Taylor, N., \& Zappi, D. (2004). Cacti of eastern Brazil. Royal BotanicGardens, 61 (2-3), 198-201. 10.1017 / S0960428605280277.

Zanine, A. M., \& Santos, E. M. (2004). Competição entre espécies de plantas - uma revisão. Revista da FZVA, 11 (1), 10-30. https://www.academia.edu/14762965/Competi\%C3\%A7\%C3\%A3o_entre_esp\%C3\%A9cies_de_plantas_uma_revis\%C3\%A3o. 